\title{
Small Scale Intramolecular Flexibility in ${ }^{111 \mathrm{~m}} \mathrm{Cd}-\mathrm{Plastocyanin} *$
}

W. Tröger a , C. Lippert ${ }^{\text {a }}$, T. Butz ${ }^{\text {a }}$, K. Sigfridsson ${ }^{b}$, Ö. Hansson ${ }^{b}$, E. McLaughlin ${ }^{c}$, R. Bauer ${ }^{\mathrm{c}}$, E. Danielsen ${ }^{\mathrm{d}}$, L. Hemmingsen $^{\mathrm{d}}$, M. J. Bjerrum ${ }^{\mathrm{d}}$, and the ISOLDE Collaboration ${ }^{\mathrm{e}}$

${ }^{a}$ Fakultät für Physik und Geowissenschaften, Universität Leipzig, Germany

b Department of Biochemistry and Biophysics, Chalmers Technical University and Göteborg University, Sweden

c Department of Biochemistry, University of Cambridge, United Kingdom

d Department of Mathematics and Physics, Royal Veterinary and Agricultural University, Frederiksberg, Denmark

e CERN, Geneva, Switzerland

Z. Naturforsch. 51 a, 431-436 (1996); received October 20, 1995

The effect of mutations in the vicinity of the putative electron transfer path on the metal center of the electron transfer protein plastocyanin (spinacea) is investigated by monitoring the nuclear quadrupole interaction of ${ }^{111 \mathrm{~m} C d}$ in Cd-derivatives of the protein via time differential perturbed angular correlation. The spectra for the wild type protein and the mutants were rather similar. All spectra exhibit a peculiar line profile which points towards a small scale intramolecular flexibility of the metal center.

Key words: TDPAC, Nuclear quadrupole interaction, ${ }^{111 \mathrm{~m} C d-P l a s t o c y a n i n, ~ f l e x i b i l i t y ~}$

\section{Introduction}

Plastocyanin (Pc) is a small (between 97-104 aminoacids, molecular weight $\approx 10 \mathrm{kD}$ ) copper-binding protein which acts as an electron carrier between the cytochrome $\mathrm{b}_{6} f$ and photosystem I complex in the photosynthetic electron transfer chain (for reviews see: [1-3]). Due to its spectroscopic properties, especially its strong optical absorbance around $600 \mathrm{~nm}$ and the associated intense blue colour, it is referred to as a blue copper protein. Other members of this family of small blue copper proteins are e.g. azurin, stellacyanin and amicyanin [4]. These proteins owe their strong blue colour to the characteristic coordination geometry of the $\mathrm{Cu}(\mathrm{II})$-site, usually denoted as "Type 1" site.

The structure of oxidized Pc (populus nigra) was determined by Guss and Freeman [5]. Copper is coordinated by two histidines (His $37, \mathrm{His} 87, \mathrm{Cu}-\mathrm{N}$ bonds), one cysteine (Cys 87, $\mathrm{Cu}-\mathrm{S}$-bond) and a methionine (Met 92, Cu-S-bond). Whereas the two nitrogens (His 37, His 87), the sulfur (Cys 84), and copper are almost in a plane with typical bond lengths between $2.0 \AA$ and $2.1 \AA$, a longer axial approach

\footnotetext{
* Presented at the XIIIth International Symposium on Nuclear Quadrupole Interactions, Providence, Rhode Island, USA, July 23-28, 1995.

Reprint requests to Prof. Dr. T. Butz.
}

(bond length $2.9 \AA$ ) is made by sulfur of the Met 92 . Since the $\mathrm{Cu}-\mathrm{S}-\mathrm{Met} 92$ distance is quite long, the involvement of Met 92 in binding to copper is difficult to assess. Only the metal ligand His 87 is exposed to the surface of the protein.

$\mathrm{Pc}$ has two potential binding sites on the surface for reaction partners. One of these sites is the hydrophobic patch around the $\mathrm{Cu}$-ligand His 87 [1]. Plastocyanin mutants in which the amino acid leucine (L12) in the vicinity of the hydrophobic patch is replaced by the amino acids alanine (A), asparagine $(\mathrm{N})$, and glutamine (E) via site directed mutagenesis [6] show a drastically reduced affinity to the photosystem I compared to the native protein [6].

We replaced isomorphously $\mathrm{Cu}$ by ${ }^{111 \mathrm{~m}} \mathrm{Cd}$ in native plastocyanin (spinacea) and in the mutants mentioned above to study the influence of these mutations on the metal site by monitoring the ${ }^{111 \mathrm{~m}} \mathrm{Cd}$-nuclear quadrupole interaction via time differential perturbed angular correlation.

\section{Experimental Methods}

Wild type Pc (spinacea) was used from two different provenances (Chalmers, Cambridge). The overexpression of Pc in E. coli, the site directed mutagenesis, and protein purification steps are described in detail in [6]. 


\begin{tabular}{lllllll}
\hline Protein & $\begin{array}{l}\omega_{1} \\
{[\mathrm{Mrad} / \mathrm{s}]}\end{array}$ & $\eta_{1}$ & $\begin{array}{l}\text { rel.frac. } \\
{[\%]}\end{array}$ & $\begin{array}{l}\omega_{2} \\
{[\mathrm{Mrad} / \mathrm{s}]}\end{array}$ & $\eta_{2}$ & $\begin{array}{l}\text { rel.frac. } \\
{[\%]}\end{array}$ \\
\hline $\operatorname{Pc}(\mathrm{wt}){ }^{*}$ & $420(2)$ & $0.427(10)$ & $36(9)$ & $420(2)$ & $0.469(7)$ & $64(9)$ \\
$\operatorname{Pc}(\mathrm{wt})^{* *}$ & $420(2)$ & $0.421(8)$ & $44(8)$ & $420(2)$ & $0.470(7)$ & $56(8)$ \\
$\operatorname{Pc}(\mathrm{L} 12 \mathrm{~A})$ & $426(3)$ & $0.444(12)$ & $28(12)$ & $425(2)$ & $0.490(7)$ & $72(12)$ \\
$\operatorname{Pc}(\mathrm{L} 12 \mathrm{E})$ & $418(3)$ & $0.431(10)$ & $70(16)$ & $418(5)$ & $0.487(16)$ & $30(16)$ \\
$\operatorname{Pc}(\mathrm{L} 12 \mathrm{~N})$ & $416(2)$ & $0.438(9)$ & $20(5)$ & $406(1)$ & $0.565(5)$ & $80(5)$ \\
\hline
\end{tabular}

Table 1 . The fitted hyperfine parameters for Pc and its mutants. The $V_{z z}-\eta$-distribution is modelled by two closely lying NQI's. The conversion from the precession frequency $\omega$ and $\eta$ into $v_{\mathrm{Q}}=e Q V_{z z} / h$ is described in [9].

* From Cambridge. ** From Chalmers.

The copper was removed from the proteins by dialysis as described in [7].

The ${ }^{111 \mathrm{~m}} \mathrm{Cd}$ activity was supplied by the mass separator ISOLDE/CERN at Geneva: ${ }^{111 \mathrm{~m}} \mathrm{Cd}$ from the radioactive beam was implanted into ice (from Milli$\mathrm{Q}$ plus water) at about $100 \mathrm{~K}$. The ice was thawed $(100 \mu \mathrm{l})$ and then added directly to the apo-proteins $(100 \mu \mathrm{l}$, protein concentration $3 \mathrm{mg} / \mathrm{ml}$ ) in $0.1 \mathrm{M}$ TRIS buffer at $\mathrm{pH}=7.2$. No inactive $\mathrm{Cd}$ was added to avoid unbound $\mathrm{Cd}$. After an incubation time of about 30 min sucrose was added with $57 \mathrm{wt} \%$ in order to immobilize the proteins. All the preparation steps with proteins were performed at $0^{\circ} \mathrm{C}$. We also carried out experiments with ${ }^{111 \mathrm{~m}} \mathrm{Cd}$ obtained by the cyclotron reaction ${ }^{108} \mathrm{Pd}(\alpha, \mathrm{n}){ }^{111 \mathrm{~m}} \mathrm{Cd}$ at the Niels Bohr Institute, Copenhagen, chemically separated from the Pd-target [7]. In this case, experiments were performed with and without carrier.

The TDPAC experiments were carried out using the high efficiency TDPAC-Camera [8] equipped with $\mathrm{BaF}_{2}$-scintillators to achieve a time resolution of about 750 ps FWHM at ${ }^{111 \mathrm{~m}} \mathrm{Cd}$ energies. The $\mathrm{pH}$ of the TDPAC samples ranged between 7.2 and 7.5 , the measurement temperature was $0^{\circ} \mathrm{C}$.

\section{Results and Discussion}

The ${ }^{111 \mathrm{~m}} \mathrm{Cd}$-TDPAC spectra and their Fourier transforms are shown in Figure 1. Obviously, the spectra for the wild type plastocyanin and its mutants are rather similar, Pc (L12N) being the only system with obvious differences to the other systems. All Fourier transforms show a peculiar line profile of a relatively sharp fundamental with broadened harmonics (Figure 1, right). These line profiles suggest a correlated distribution of the largest component of the electric field gradient tensor (EFG) $V_{z z}$ and the asymmetry parameter $\eta=\left(V_{x x}-V_{y y}\right) / V_{z z}$. This correlated distribution was modelled by two closely lying NQI's in the least squares fitting analysis (see Table 1).

In order to unravel this correlated distribution we performed a cross-correlation analysis [10]. In this type of analysis the experimental Fourier spectrum is cross-correlated with a theoretical spectrum for all possible values of $v_{\mathrm{Q}}$ and $\eta$. The results are displayed in Fig. 2 for a simulated spectrum with instrumental linewidth, i.e. no distribution of $V_{z z}$ and $\eta$, and for Pc (wt) and its mutants. In this type of plot, the co-called Czjzek-plot [11], which is shown in Fig. 3, lines of constant $V_{z z}$ (or $v_{\mathrm{Q}}=e Q V_{z z} / h$ ) are straight lines which are perpendicular to the upper boundary. In fact, both axes are in units of $e Q / h$. In this way, the ordinate gives directly $v_{\mathrm{Q}}$ at the $\eta=0$ boundary. Lines of constant $\eta$ are straight lines ranging from the upper boundary $(\eta=0)$ down to the horizontal $(\eta=1)$. It is immediately obvious that in Pc and its mutants the $V_{z z}-\eta$-spot, which is roughly circular in the simulated spectrum (see Fig. 2 a), is elongated along the trajectory (i.e. the line of constant line position in the Fourier spectrum) for the fundamental frequency which is forming an angle of roughly $60^{\circ}$ with the horizontal. This correlated $V_{z z}-\eta$-distribution is characteristic for trigonal in-plane flexibility in planar geometries.

The argument is as follows: suppose, the charge density distribution around the central atom in a planar geometry can be split into a radial part and an angular part; furthermore, the radial part is assumed to be constant while the bond angles vary. In this situation, one of the EFG tensor components is perpendicular to the plane due to symmetry and does not depend on the bond angles of the ligand, i.e. it is constant. For a certain range of bond angles, this EFG tensor component happens to be the largest component (in magnitude), $V_{z z}$, and forms a straight line inclined at $60^{\circ}$ in the Czjzek-plot [11] (see Figure 3). This line extends linearly into the upper half $\left(V_{z z}>0\right)$, 

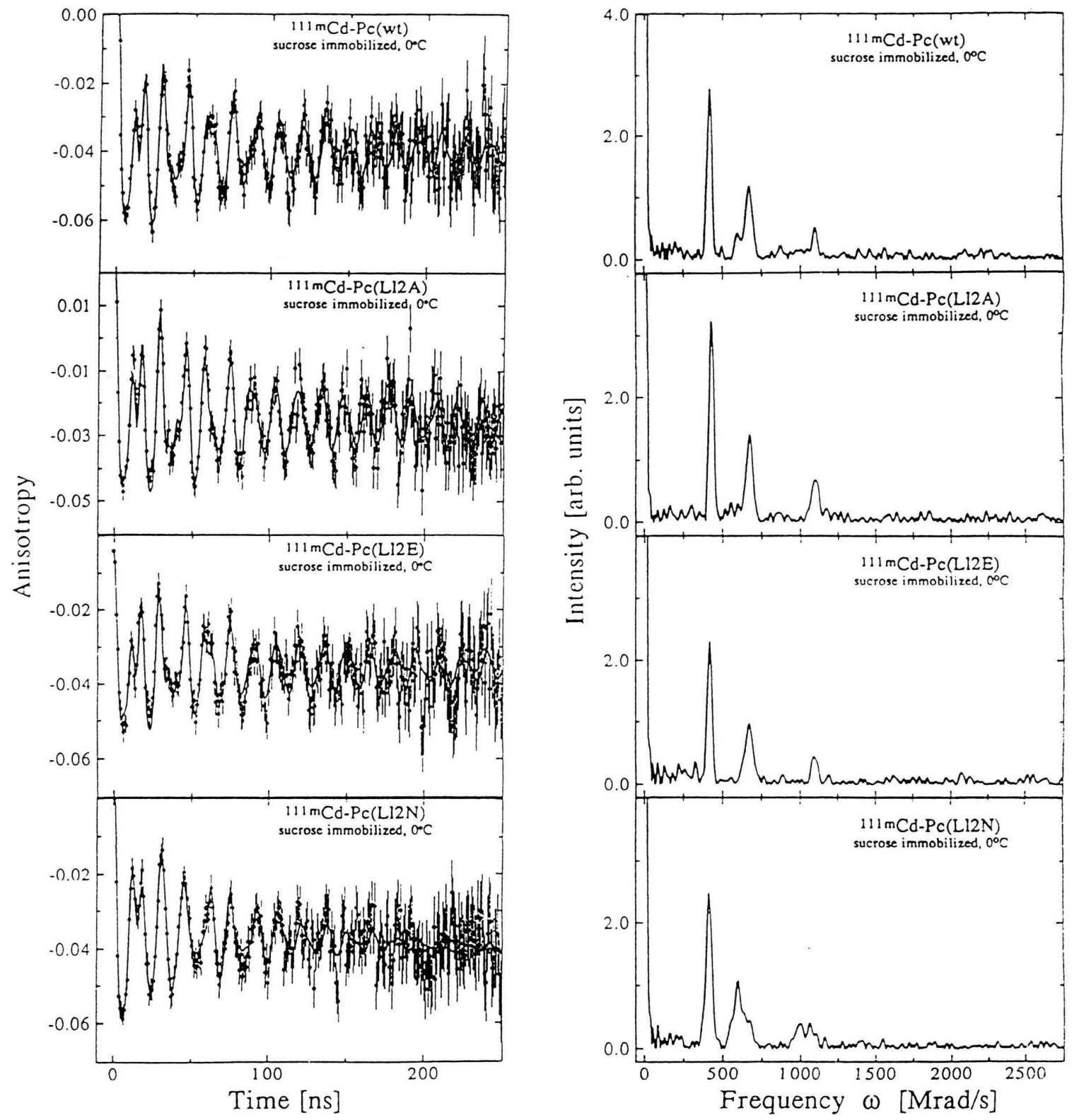

Fig. 1. TDPAC spectra and their Fourier transforms of the ${ }^{111 \mathrm{~m}} \mathrm{Cd}$-derivatives of the wildtype plastocyanin and the plastocyanin mutants $\operatorname{Pc}(\mathrm{L} 12 \mathrm{~A})$. Pc(L12E), and $\mathrm{Pc}(\mathrm{L} 12 \mathrm{~N})$.

where both $V_{z z}$ and $\eta$ vary with bond angles. Here $V_{y y}$ (or $V_{x x}$ ) is constant. This follows from the fact, that the non-constant EFG-tensor components depend linearly on each other due to the fact that the trace of the EFG-tensor must be zero. Such a tensor can be written after diagonalization as

$$
\left(\begin{array}{ccc}
-1 / 2 & 0 & 0 \\
0 & -1 / 2 & 0 \\
0 & 0 & 1
\end{array}\right)+\alpha\left(\begin{array}{ccc}
1 & 0 & 0 \\
0 & -1 & 0 \\
0 & 0 & 0
\end{array}\right)
$$

where $\alpha$ contains all angular information. This is a consequence of the structure of the quadratic equation 

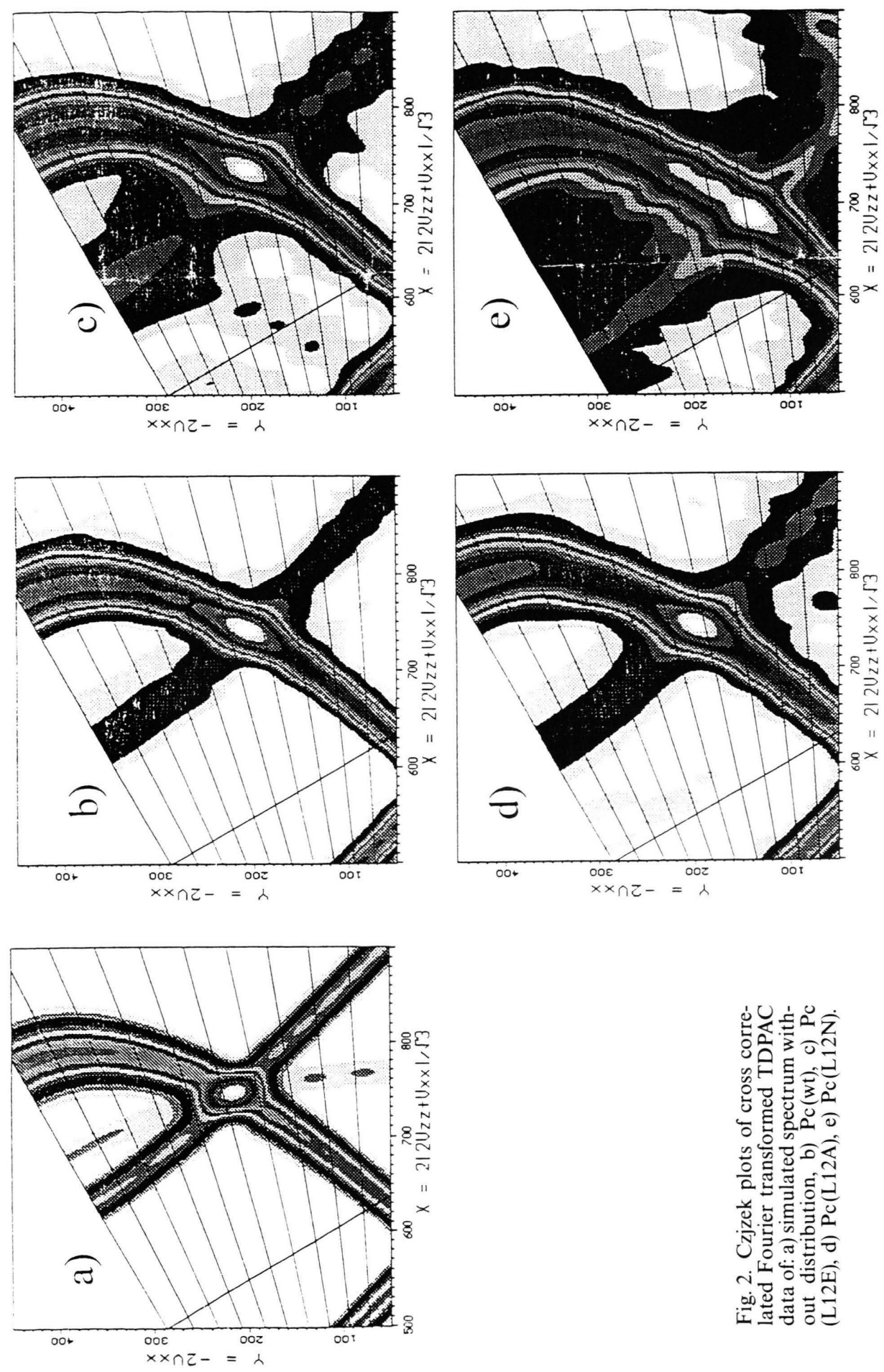


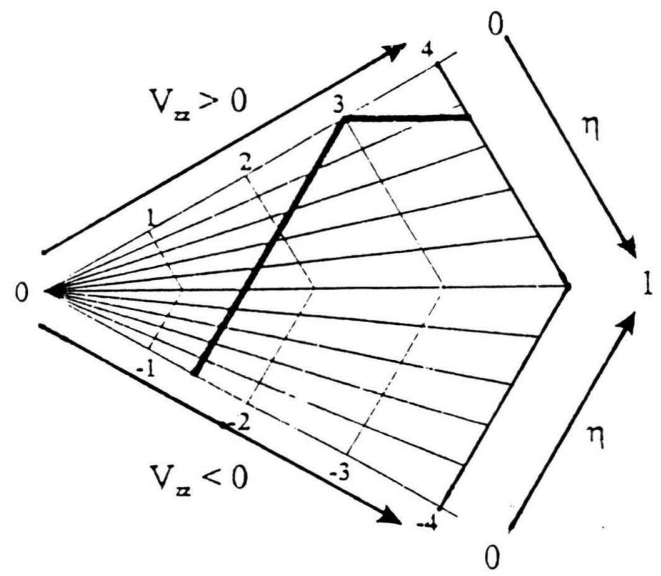

Fig. 3. Czjzek-plot. The bold line represents the trajectory describing torsions in planar geometry.

which has to be solved in order to diagonalize the tensor. The trajectory corresponding to this tensor for variable $\alpha$ is shown as a bold line in Figure 3. The lower endpoint at $\eta=0$ correspond to a geometry with axial symmetry and $V_{z z}$ pointing perpendicular to the plane. For three identical ligands, e.g., this would be a trigonal geometry with $120^{\circ}$ ligand-metal-ligand bond angles. Any deformation of this geometry would increase $\eta$ at constant $V_{z z}$ until at $\eta=1$ the sign changes and $V_{z z}$ now flips to an in-plane orientation. The upper endpoint at $\eta=0$ corresponds to a collinear geometry with $V_{z z}$ being parallel to this line. Of course, spatial constraints would restrict the accessible range of this trajectory. In particular, the horizontal portion of the trajectory is never accessible. For a three-fold planar coordination, with a dominating contribution to the EFG from the cysteine sulfur, as deducd for azurin [7], $V_{z z}$ is in the plane and $V_{y y}=$ const. Due to the similarity of the NQI parameters between azurin and plastocyanin we believe that the same situation prevails. It should be mentioned that we did not determine the sign of $V_{z z}$. Therefore we arbitrarily placed the $V_{z z} / \eta$-pair for Pc in the upper half of the Czjzekplot. The essential feature in the Czjzek-plot (Fig. 2) is the fact that for sufficiently large $\eta$-values the line of constant fundamental line position in the Fourier spectrum forms roughly an angle of $60^{\circ}$ with the horizontal, i.e. it is parallel to the trajectory for planar geometries. Hence any angular variation in this geometry will stretch the $V_{z z}, \eta$-spot along this trajectory.

One might argue that the $\mathrm{Cu}$-replacement by $\mathrm{Cd}$ induces the small scale intramolecular in-plane flexibility and that this is not characteristic for the biologically active $\mathrm{Cu}$-protein. However, we believe that this is not true. In fact, $\mathrm{Pc}$ is the only native protein where such a distribution of $V_{z z}$ and $\eta$ is observed. Other native proteins such as azurin, stellacyanin [7] or the multi-copper proteins ascorbate oxidase and laccase [12] do not exhibit such distributions. Admittedly, we frequently observe two slightly inequivalent coordination geometries for azurin M121 mutants judging from the splitting of Fourier peaks into double peaks [7]. It seems that any modification of the native protein leads to small scale intramolecular bistabilities as revealed by the ${ }^{111 \mathrm{~m}} \mathrm{Cd}-\mathrm{NQI}$. However, the important feature in $\mathrm{Pc}$ is the fact, that even the native protein seems to lack a unique coordination geometry on a $\pm 2^{\circ}$ angular variation level. An estimate of this sensitivity can be obtained using a point charge model. This flexibility is possibly related to the changes in coordination geometry as a function of $\mathrm{pH}$, as revealed by $\mathrm{X}$-ray crystallography for reduced $\mathrm{Cu}(\mathrm{I})$ plastocyanin [13]. It should be mentioned that $\mathrm{Cd}^{2+}$ has a $\mathrm{d}^{10}$-electron configuration. Whether this flexibility is of functional importance or not remains speculation at present.

In conclusion, TDPAC is a very sensitive tool to reveal intramolecular instabilities in macromolecules via the detection of NQI's.

\section{Acknowledgement}

We are grateful to $\mathrm{S}$. Young for help with cultivation and purification of the plastocyanin mutants. We gratefully acknowledge the assistance of Th. Beyer during the ISOLDE beam times. It is a pleasure to thank D. Forkel-Wirth, S. G. Jahn, and J. G. Correia of the ISOLDE Collaboration for their help with the activity collection. We appreciate the help of $\mathrm{M}$. A Taylor and P. Ganal in preparing the figures. This work was supported by the Deutsche Forschungsgemeinschaft (Bu 594/5, Schwerpunktprogramm: "Bioanorganische Chemie: Übergangsmetalle in der Biologie und ihre Koordinationschemie"). 
[1] A. G. Sykes, Structure and Bonding 78, 175 (1991).

[2] E. L. Gross, Photosynthesis Research 37, 103 (1993).

[3] M. R. Redinbo, T. O. Yeates, and S. Merchant, Journal of Bioenergetics and Biomembranes 26, 49 (1994).

[4] E. T. Adman, "Structure and Function of Small Blue Copper Proteins" in "Metalloproteins", Part 1: "Metal Proteins with Redox Roles", Ed. P. Harrison, Verlag Chemie, 1985.

[5] J. M. Guss and H. C. Freeman, J. Mol. Biol. 169, 521 (1983).

[6] M. Nordling, K. Sigfridsson, S. Young, L. G. Lundberg, and Ö. Hansson, FEBS 291, 327 (1991) and references therein; K. Sigfridsson, S. Young, and Ö. Hansson, to be published.

[7] E. Danielsen, R. Bauer, L. Hemmingsen, M.-L. Anderson, M. B. Bjerrum, T. Butz, W. Tröger, G. W. Canters, C. W. G. Hoitink, G. Karlsson, Ö. Hannson, and A. Messerschmidt, J. Biol. Chem. 270, 573 (1995).
[8] T. Butz, S. Saibene, Th. Fraenzke, and M. Weber, Nucl. Instrum. Meth. A 284, 417 (1989).

[9] T. Butz, Hyp. Int. 52, 189 (1989).

[10] T. Butz, M. Ceolín, P. Ganal, P. Schmidt, M. A. Taylor, and W. Tröger, to be published in "Proceedings of the Xth International Conference on Hyperfine Interactions", Leuven, 28.8.-1.9.95.

[11] G. Czjzek, Hyperf. Int. 14, 189 (1983).

[12] T. Butz, W. Tröger, A. Messerschmidt, U. Thoenes, and R. Huber, Hyp. Int. 80, 1127 (1993).

[13] J. M. Guss, P. R. Harowell, M. Murata, V. A. Norris, and H. C. Freeman, J. Mol. Biol. 192, 361 (1986). 